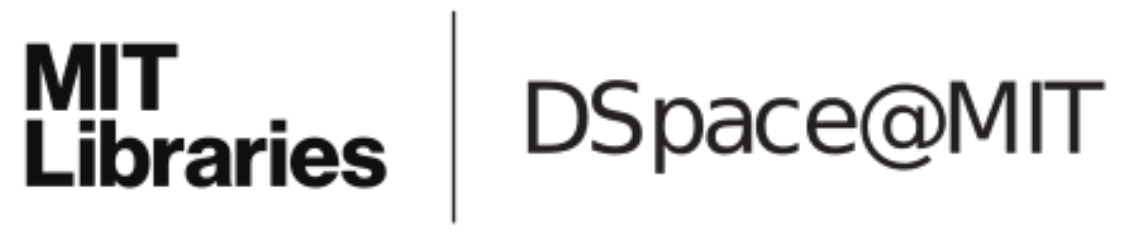

\author{
MIT Open Access Articles
}

\section{Analyzing the dynamics of brain circuits with temperature: Design and implementation of a miniature thermoelectric device}

The MIT Faculty has made this article openly available. Please share how this access benefits you. Your story matters.

Citation: Aronov, Dmitriy, and Michale S. Fee. "Analyzing the Dynamics of Brain Circuits with Temperature: Design and Implementation of a Miniature Thermoelectric Device." Journal of Neuroscience Methods 197, no. 1 (April 2011): 32-47.

As Published: http://dx.doi.org/10.1016/j.jneumeth.2011.01.024

Publisher: Elsevier

Persistent URL: http://hdl.handle.net/1721.1/102252

Version: Author's final manuscript: final author's manuscript post peer review, without publisher's formatting or copy editing

Terms of use: Creative Commons Attribution-Noncommercial-NoDerivatives 


\title{
Analyzing the dynamics of brain circuits with temperature: design and implementation of a miniature thermoelectric device
}

\author{
Dmitriy Aronov and Michale S. Fee \\ McGovern Institute for Brain Research, Department of Brain and Cognitive Sciences, \\ Massachusetts Institute of Technology, MIT 46-5130, 77 Massachusetts Ave, Cambridge, MA \\ 02139
}

\section{Introduction}

Complex behaviors require brain circuits that evolve in time through distinct sequences of states (Lashley, 1951; Tanji, 2001). When we speak a word, or play the piano, the appropriate muscles are activated in a highly coordinated temporally precise pattern (Levelt, 1993). This temporal pattern of muscle activation is driven by motor neurons, which in turn can be activated by temporally patterned activity in local spinal interneurons, midbrain motor systems and descending cortical pyramidal tract neurons. But where does temporal structure originate? Where are the biophysical processes that drive such temporal evolution of brain activity?

In some simple nervous systems, it has been possible to isolate small circuits of neurons containing the biophysical dynamics that generate the temporal pattern of a behavior. For example, Stent et al (1978) describe an oscillator network that generates the underlying oscillatory rhythm of swimming behavior in the leech. Neurons in the oscillator network can be distinguished from other neurons in the network that might be referred to as 'follower' neurons. Follower neurons are necessary for swimming, but their activity is driven by the oscillator network, and they are not involved in the generation of the underlying temporal structure of the behavior. Here we say that the 'biophysical dynamics' that underlie the swimming behavior are located entirely within the oscillator network, even though the follower neurons also exhibit time-dependent activity associated with swimming.

Are there similar specialized circuits in the brain that generate the temporal structure underlying complex mammalian behaviors? Do different brain regions control different timescales or hierarchical structures of a behavior (such as phonemes and words in speech)? Similar questions can be asked about the origin of internal dynamics of the brain. For example, which neurons or networks of neurons express biophysical dynamics that produce particular types of oscillations (Buzsáki, 2006) or neuronal sequences (Abeles, 1991; Hahnloser et al., 2002; Pastalkova et al., 2008)?

Questions about brain dynamics are difficult to address using standard techniques of electrophysiological recording and inactivation. These methods tell us whether a brain

\footnotetext{
(C) 2011 Elsevier B.V. All rights reserved.

*To whom correspondence should be addressed: fee@ mit.edu, (617) 324-0173 (phone), (617) 324-0256 (fax).
}

Publisher's Disclaimer: This is a PDF file of an unedited manuscript that has been accepted for publication. As a service to our customers we are providing this early version of the manuscript. The manuscript will undergo copyediting, typesetting, and review of the resulting proof before it is published in its final citable form. Please note that during the production process errors may be discovered which could affect the content, and all legal disclaimers that apply to the journal pertain. 
region is involved in the expression of a particular behavior, but they do not reveal whether the biophysical dynamics within that region are actively involved in timing the behavior. For example, in the songbird, electrophysiological recordings reveal that temporally patterned activity related to a song sequences may be found in several brain regions ( $\mathrm{Yu}$ and Margoliash, 1996; Hahnloser et al., 2002), and muscles (Goller and Cooper, 2004); in most cases, however, these sequences are not generated by the neurons that express them, but are driven synaptically by upstream brain areas (Hahnloser et al., 2002; Long and Fee, 2008). The most direct way to localize the dynamics is to manipulate the biophysics in different regions and observe the resulting effect on behavior or network activity. Such direct manipulation of neuronal dynamics can be accomplished using intracellular current injection (Stent et al., 1978; Bonifazi et al., 2009), by pharmacological manipulations (Harris-Warrick and Cohen, 1985; Tryba et al., 2008), or with temperature changes (Arbas and Calabrese, 1984; Bauer and von Helversen, 1987; Pires and Hoy, 1992; Katz et al., 2004; Yamaguchi et al., 2008; Long and Fee, 2008).

Temperature affects the speed of all cellular processes, including synaptic transmission, action potential generation, and axonal conduction (Thompson et al., 1985; Volgushev et al., 2000a, 2000b). This approach is therefore broadly applicable, allowing one to slow down or speed up the dynamics of individual brain regions. Temperature modulation is, in fact, effective at changing the speeds of simple, rhythmic behaviors that are driven by central pattern generators (CPGs), such as the heart-beat in leech (Arbas and Calabrese, 1984), wing-beat in locusts (Foster and Robertson, 1992), locomotion in the Tritonia mollusk (Katz et al., 2004), stomatogastric rhythm in the crab (Tang et al., 2010), as well as communication signals in fish (Feng, 1976), insects (Bauer and von Helversen, 1987; Pires and Hoy, 1992), and frogs (Gerhardt, 1978; Yamaguchi et al., 2008). Furthermore, experimentally focusing temperature changes to specific regions of the nervous system is effective at localizing the biophysical processes that produce these motor rhythms (Bauer and von Helversen, 1987; Pires and Hoy, 1992).

Recent work indicates that this technique can be extended to the study of more complex behaviors in the vertebrate brain. In zebra finches, cooling the forebrain nucleus HVC (proper name, formerly the high vocal center) changes song speed uniformly across all timescales, whereas temperature changes in its efferent nucleus RA (robust nucleus of the arcopallium) have no effect on song timing (Long and Fee, 2008). These findings suggest that the dynamics intrinsic to $\mathrm{HVC}$ are actively involved in the control of song timing; if song timing was generated entirely upstream of HVC, cooling HVC could disrupt song acoustic structure, but would not change its temporal structure.

Cooling of brain structures in small, behaving, warm-blooded animals poses several technological challenges. For instance, probes that use liquid or gas flow for cooling are generally not lightweight or flexible enough for this application (Zhang et al., 1986; Lomber et al., 1999). Alternatively, commercially-available Peltier thermoelectric devices have been used to cool parts of the brain, but these also require water flow to remove excess Ohmic heat generated by electric currents (Ferster et al., 1996; Chafee and Goldman-Rakic, 2000; Imoto et al., 2006). Recently, a Peltier device that uses water cooling has been miniaturized to be carried by a small songbird (Long and Fee, 2008). For some applications, however, it is desirable to eliminate the water cooling system, which partially restrains the animal with attached tubing. Another challenge is that cooling deep brain structures requires efficient heat transfer across long distances in the brain - a process opposed by the animal's homeostatic temperature maintenance.

Here, we discuss the design, optimization and implementation of a lightweight $(<1 \mathrm{~g})$ Peltier thermoelectric device useful for modulating temperature in brain structures of small animals. 
We present two versions of the device: one used for cooling superficial regions by placing cooling pads on the surface of the brain (Fig. 1A-C), and another used for cooling deep regions via insulated, thermally conductive probes implanted into the brain (Fig. 1D-F). For structures in the forebrain song system of the zebra finch, the devices we built are capable of generating continuous cooling by any temperature up to $\sim 7^{\circ} \mathrm{C}$ below body temperature - an amount sufficient for manipulating biophysical dynamics (Long and Fee, 2008). The principles of this method are generally applicable for constructing devices that use temperature to probe circuit dynamics in behaving animals.

\section{Theoretical Methods: A simple steady-state model for thermoelectric devices}

In order to properly analyze and optimize the construction of a thermoelectric device for brain cooling, it is necessary to have a simple mathematical framework for describing the operation of such a device. Here we describe such a framework, including the effect of cooling probes on brain tissue. Equations described in the following sections are also available in the computer code in Supplementary Material.

\subsection{Basics of thermoelectric devices}

When electric current is passed across the junction between a metal and a semiconductor, it causes heat to be pumped across the junction. This phenomenon - known as the Peltier effect - is the basis of thermoelectric devices (Rowe, 1995). If the semiconductor is doped with positively charged ions (p-type), then the flow of electrons from the semiconductor to the metal causes heat to be pumped from the semiconductor to the metal as well. On the contrary, if the semiconductor is doped with negatively charged ions (n-type), then heat is pumped in the opposite direction. P- and n-type semiconductors can be cleverly alternated between two plates (Fig. 2A), so that heat is pumped from one plate (the cold plate) and into another (the hot plate) as current flows back and forth through the device. At each junction, the net amount of heat pumped is given by $Q=\alpha T I$, where $T$ is the absolute temperature of the junction, $I$ is the amount of current, and $\alpha$ is a property of the semiconductor material known as the Seebeck coefficient. (In this formula, $\alpha$ is the average absolute value of the coefficients for the $\mathrm{n}$ - and p-type elements, which can be different.)

Unfortunately, two phenomena limit the efficiency of this arrangement: First, semiconductors are, by definition, not perfect electrical conductors, and the flow of current through them causes a dissipation of heat. It can be shown that half of this Ohmic heat flows into the hot plate and the other half into the cold plate. Combined with the Peltier effect, the net amount of heat pumped into the cold plate is therefore

$$
\dot{Q}_{C}=2 N\left(a T_{C} I+\frac{1}{2} R I^{2}\right)
$$

where $2 N$ is the number of semiconductor elements (forming $N$ junctions), $T_{C}$ is the absolute temperature of the cold plate, and $R$ is the electrical resistance of each semiconductor element. Note that the sign of the first term in Eqn. 1 is chosen such that, by convention, negative current causes cooling at the cold plate. Similarly, the net amount of heat pumped into the hot plate is

$$
\dot{Q}_{H}=2 N\left(-a T_{H} I+\frac{1}{2} R I^{2}\right)
$$


where $T_{H}$ is the absolute temperature of the hot plate. For mild cooling applications, the first term in Eqn. 2 is close in magnitude to the first term in Eqn. 1 (since absolute temperatures $T_{C}$ and $T_{H}$, in Kelvin, are numerically close). ${ }^{1}$ Thus, we can roughly think of the first terms in these expressions as representing the pumping of heat out of the cold plate and into the hot plate. The second term represents Ohmic heat that flows into both plates. For negative values of $I$, the two effects produce heat flow in opposite directions at the cold plate; thus, Ohmic heating reduces the effectiveness of cooling.

A second problem is that the semiconductor elements between hot and cold plates can have substantial thermal conductivity, allowing heat to flow back from the hot plate to the cold plate, also reducing the effectiveness of cooling. A simple expression can be used to quantify the amount of heat that flows back:

$$
\dot{Q}_{s}=-2 N K \Delta T
$$

in terms of the thermal conductance $K$ of each semiconductor element and the temperature difference $\Delta T=T_{C}-T_{H}$ between the two plates

\subsection{Equivalent circuit for analyzing device performance}

By analogy with electrical circuits, we can represent semiconductors as a thermal resistance (inverse of thermal conductance) connecting the two plates (Fig. 2B). In this circuit, temperature is treated analogously to voltage in an electrical circuit and heat transfer is treated analogously to current. The relationships between these thermal quantities follows a relation analogous to Ohm's Law in electrical circuits (for example, Eqn. 3).

The hot plate is usually connected to the external world through a thermal conductor called the heat sink. In the simplest, ideal scenario the heat sink is perfect - i.e., by analogy with electrical circuits, the hot plate is "grounded" (Fig. 2B). In this case, the hot-plate temperature remains at a constant value, whereas the cold-plate temperature is changed by an amount $\Delta T$ relative to this value. In the following sections, we investigate the dependence of $\Delta T$ on the amount of electrical current $I$ in this ideal case; subsequently we analyze the effects of imperfect heat sinking on device performance.

\subsection{Thermal and electrical properties of the semiconductor elements}

Three properties of the semiconductor elements are present in Eqns. 1 through 3 and therefore affect heat transfer in the Peltier device. These are the Seebeck coefficient $\alpha$, the electrical resistance $R$, and the thermal conductance $K$. The latter two quantities depend on the shape of a semiconductor element in addition to its material properties. For a semiconductor block of height $h$ and cross-sectional area $A$, we can define the geometric constant $G=A / h$. Electrical resistance of this block is then given by $R=\rho / G$ and thermal conductance by $K=\kappa G$, where $\rho$ is the electrical resistivity of the material and $\kappa$ is the thermal conductivity. These equations suggest that changing $G$ can have opposing effects on the efficiency of the device: whereas increasing $G$ reduces Ohmic heating due to the effect on $R$, it also reduces thermal isolation between the hot and the cold plate due to the effect on $K$. The principles behind choosing an optimal value of $G$ will be discussed later.

\footnotetext{
${ }^{1}$ We limit analytical treatment of the device to small temperature changes $(\sim 10 \mathrm{~K}$ from a baseline of $\sim 314 \mathrm{~K})$ because these are sufficient for mild brain cooling applications, but also because semiconductor properties are temperature-dependent and cannot be treated as constants in the general case. For applications that require stronger cooling, dynamical simulation of the device using empirical measurements of semiconductor properties is required for accurate analysis (Kondratiev and Yershova, 2001).
} 


\subsection{Behavior of an isolated device}

In steady state and in the absence of thermal conductances linking the device to the external world, heat transfers given by Eqns. 1 and 3 must add to zero at the cold plate. That is, heat transfer away from the cold plate due to the Peltier effect must be equal to the heat transfer into the cold plate due to Ohmic heating and thermal diffusion:

$$
2 N \alpha T_{C} I+\frac{N \rho}{G} I^{2}-2 N \kappa G \Delta T=0
$$

Although $T_{C}$ is not independent of $\Delta T$, for mild brain cooling experiments these values are very different numerically $\left(|\Delta T| \ll T_{C}\right)$. When solving for the steady-state value of $\Delta T$, we can therefore approximate $T_{C}$ as a constant value $T$. (In simulations discussed later, we approximate $T$ as being roughly equal to the body temperature $T_{B}$; see Table 1 ). Given this approximation, the solution to Eqn. 4 is

$$
\Delta T=\frac{\alpha T}{\kappa G} I+\frac{\rho}{2 \kappa G^{2}} I^{2}
$$

This concave-up quadratic form implies the existence of a minimum temperature at the cold plate at some "optimal" amount of current (Fig. 2C). Beyond this amount of current, marginal increases in Ohmic heating are greater than the marginal increases in Peltier cooling (since the former is quadratic in current, whereas the latter is only linear). Optimizing Eqn. 5 to find the minimum achievable steady-state $\Delta T$, we find the optimum current $I_{\mathrm{opt}}$ :

$$
I_{\mathrm{opt}}=-\frac{\alpha G T}{\rho}
$$

The temperature difference $\Delta T_{\text {opt }}$ at this current is

$$
\Delta T_{\mathrm{opt}}=\frac{\alpha^{2} T^{2}}{2 \kappa \rho}=Z \frac{T^{2}}{2}
$$

where the quantity $Z=\alpha^{2} / \kappa \rho$ is a figure of merit for the semiconductor material (Rowe, 1995).

\subsection{Behavior of the device attached to a load}

In practice, the purpose of a thermoelectric device is to pump heat from a thermal load (in our case, the brain). By analogy to electrical circuits, the load in steady-state can be represented by a thermal conductance $K_{L}$ connecting the cold plate to "ground." The ground in a thermal circuit is a fixed temperature, relative to which cooling is measured; in the case of brain cooling, it is the normal temperature of the brain, $T_{B}$ (about $314 \mathrm{~K}$ in zebra finches; (Bech and Midtgård, 1981)).

Introducing the load as a conductor to the thermal circuit (Fig. 2D), we can solve for the steady-state temperature as a function of current: 


$$
\Delta T=\frac{\alpha T}{\kappa G+K_{L}} I+\frac{\rho}{2 \kappa G^{2}\left(1+K_{L} / 2 N \kappa G\right)} I^{2}
$$

In the case of $K_{L}=0$, this equation simplifies to Eqn. 5, derived above. Again, the relationship of temperature on current is quadratic, exhibiting an optimum. The main effect of the load is the reduction in the optimal cooling achieved at the cold plate (Fig. 2C). The optimum current is

$$
I_{\mathrm{opt}}=-\frac{\alpha G T\left(\kappa G+K_{L} / 2 N\right)}{\rho\left(\kappa G+K_{I}\right)}
$$

which simplifies to Eqn. 6 in the absence of a load, and the temperature difference at this current is

$$
\Delta T_{\mathrm{opt}}=\frac{\alpha^{2} T^{2} G\left(\kappa G+K_{L} / 2 N\right)}{2 \rho\left(\kappa G+K_{L}\right)^{2}}
$$

which simplifies to Eqn. 7 for zero load. Note that the amount of heat pumped from the load at the optimal current is $\Delta T_{\mathrm{opt}} K_{L}$.

\subsection{Behavior of the device with imperfect heat sinking}

In reality, miniature heat sinks applicable for use in small behaving animals are likely to be inefficient. We thus need to expand the above model to include imperfect heat sinks attached to the hot plate of the thermoelectric device. In the simplest case, the device is cooled convectively by a fluid (e.g. water or air). Such a heat sink can be represented by a single thermal conductance $K_{C}$ linking the hot plate to the temperature of the fluid, $\Delta T_{F}$ (measured relative to the body temperature $T_{B}$ ). This temperature is analogous to a voltage source in electrical circuits (Fig. 2E).

It is also possible to place a heat sink in direct contact with the animal's body ("bodycoupled heat sink"). Because we define the body temperature as the "ground" in our thermal circuit, this configuration can be represented by a single conductance $K_{B}$ connecting the hot plate to ground. If both convective and body-coupled heat sinks are present, the total heat sink conductance is $K_{C}+K_{B}$, and the temperature differential of the fluid can be represented by constant "bias" heat transfer $-K_{C} \Delta T_{F}$ out of the hot plate. The advantages of introducing a body-coupled heat sink to the device will be discussed later.

Treating Fig. 2E as a circuit analogous to electrical circuits, we can solve for the steady-state temperature of the cold plate:

$$
\Delta T_{C}=\frac{2 N \kappa G\left(2 N \alpha T I-N \rho I^{2} / G-K_{C} \Delta T_{F}\right)-\left(2 N \alpha I+2 N \kappa G+K_{B}+K_{C}\right)\left(2 N \alpha T I+N \rho I^{2} / G\right)}{\left(2 N \alpha I-2 N \kappa G-K_{I}\right)\left(2 N \alpha I+2 N \kappa G+K_{B}+K_{C}\right)+(2 N \kappa G)^{2}}
$$

Here, $\Delta T_{C}$ is the temperature of the cold plate relative to the brain $\left(\Delta T_{C}=T_{C}-T_{B}\right)$. Since this equation is not only demonstrative, but expresses the full model we use to characterize the device, it no longer assumes that the hot and cold plates are at a constant temperature when calculating the magnitude of the Peltier effect (see Section 3). Again, this analytic 
solution at any amount of current can be used to determine the amount of heat pumped from the brain: $\Delta T_{C} K_{L}$.

\subsection{Modeling thermal propagation in the brain}

Equations so far have represented the thermal load on the device by a single conductance $K_{L}$. In the case of brain cooling, this conductance depends on the biophysical properties of the brain tissue, as well as on the spatial properties of the contact between the device and the brain. In this section, we consider the effects of these parameters on heat propagation. The first goal of this analysis is to determine the load $K_{L}$, a quantity necessary in the above equations to quantify heat transfer from the brain. The second goal is to characterize the spatial properties of thermal propagation in the brain itself, which is necessary to understand how temperature changes as a function of distance from the device.

In some cases, the region of interest is located near the brain surface (e.g., area HVC in songbirds or superficial cortical layers in mammals), permitting temperature to be modulated by directly placing the cold plate of the device on the surface of the brain (Long and Fee, 2008). We refer to this arrangement as the planar geometry. In other cases, the region of interest is located deep in the brain, requiring that a thermally conductive probe be attached to the cold plate and placed within the brain. We call this cylindrical geometry. Our next goal is to characterize temperature changes in the brain in each of these two geometries.

2.7.1 Planar geometry-Temperature changes are the easiest to analyze in planar geometry, in which a cooling surface is placed directly in contact with the brain. In this arrangement, temperature as a function of time $t$ and distance $x$ from the surface is described by the standard one-dimensional model of thermal diffusion in solids, which includes an additional term to describe the loss of heat due to the perfusion of the brain with blood (Kastella and Fox, 1971; Jafari and Higgins, 1989):

$$
\kappa_{B} \frac{\partial^{2} \Delta T}{\partial x^{2}}-w c \Delta T-c_{B} \frac{\partial \Delta T}{\partial t}=0
$$

Here, $\kappa_{B}$ is the thermal conductivity of the brain (see Table 1), $w$ is the blood supply per units of volume and time (in $\mathrm{ml} /\left(\mathrm{cm}^{3} \cdot \mathrm{s}\right)$ ), $c$ is the specific heat capacity of the blood (in J/ $\left(\mathrm{cm}^{3} \cdot \mathrm{K}\right)$ ), and $c_{B}$ is the specific heat capacity of the brain (in $\mathrm{J} /\left(\mathrm{cm}^{3} \cdot \mathrm{K}\right)$ ). Note that temperature $\Delta T$ is measured relative to the normal brain temperature (i.e., temperature of the blood; $\Delta T=T-T_{B}$, where $T$ is the absolute temperature). In steady state, Eqn. 12 has an exponential solution $\Delta T(x)=\Delta T_{C} e^{-x / \lambda}$, where $\Delta T_{C}$ is the steady-state temperature of the cooling surface and $\lambda \equiv\left(\kappa_{B} / w c\right)^{1 / 2}$. The length constant $\lambda$ is a critical property of the brain, roughly indicating the spatial extent of temperature changes produced by the device. This solution ignores the edges of the cooling surface and is therefore only accurate at distances from the cooling plate that are less than the size of the cooling plate.

Heat flux from the brain into the cold plate is proportional to the slope of the temperature profile at the cold plate $\left(\kappa_{B} \partial \Delta T / d x\right.$ at $\left.x=0\right)$. Evaluating this quantity for the exponential solution given above, dividing by $\Delta T_{C}$, and multiplying by the surface area of the cold plate, $A_{C}$, yields the thermal conductance of the load:

$$
K_{L}=\frac{A_{C} \kappa_{B}}{\lambda}
$$


In reality, the cold plate is linked to body temperature not only via a direct contact with the brain, but also by contacting the nearby cranium through some insulation material. Because some regions of the cranium are unavoidably very close to the cold plate, and any insulation material that may be used is imperfect, this contact must be represented by a conductance $K_{I}$ (Table 1; Fig. 2F). The total load on the cold plate is therefore $K_{L}+K_{I}$, and the size of $K_{I}$ determines what fraction of the heat is pumped into the cold plate from the brain, rather than via the insulation.

2.7.2 Cylindrical geometry-When the region of interest is located deep in the brain, a thermally conductive probe must be attached to the cold plate and inserted into the brain to facilitate heat transfer (Long and Fee, 2008). We first consider how temperature in the brain changes as a function of distance from the surface of a cylindrical probe. We assume that temperature changes along the length of the probe are very gradual in comparison with radial changes in the brain. This assumption is accurate for probes made of highly conductive materials, such as silver, which is almost four orders of magnitude more conductive than the brain (see Table 1). In cylindrical coordinates, the model of thermal diffusion in perfused tissues yields the following steady-state equation for temperature (Jafari and Higgins, 1989):

$$
\frac{1}{r} \frac{d}{d r}\left(r \frac{d \Delta T}{d r}\right)-\lambda^{2} \Delta T=0
$$

where $\lambda$ is the same length constant of the brain as discussed above, $r$ is the radial distance from the center of the probe, and $\Delta T$ is the temperature change relative to normal brain temperature, assumed to be a function only of radial distance from the cylindrical probe $\left(\Delta T(r)=T(r)-T_{B}\right)$. The solution to this equation is

$$
\Delta T(r)=\Delta T_{P} \frac{K_{0}(r / \lambda)}{K_{0}\left(r_{P} / \lambda\right)}
$$

where $\Delta T_{P}$ is the steady-state temperature of the probe surface, $r_{P}$ is the radius of the probe, and $K_{0}(r)$ is the zero-order modified Bessel function of the second kind (Bowman, 1958). (Here, we used $\Delta T\left(r_{P}\right)=\Delta T_{P}$ and $\Delta T(\infty)=0$ as the boundary conditions). $K_{0}(r)$ has a steeper decrease than an exponential function at small radii; at large distances, it decreases approximately exponentially. By fitting Eqn. 15 to the data from Long and Fee (2008), we obtain an approximation of the length constant for the zebra finch forebrain, $\lambda=1.59 \mathrm{~mm}$.

In addition to characterizing the spatial profile of temperature in the brain, Eqn. 15 can be used to calculate the surface thermal conductivity of the probe-brain interface (this is conceptually similar to the electrical membrane resistivity of neuronal axons in the traditional Cable Theory; (Rall, 1962;Dayan and Abbott, 2001)). Heat transfer from the brain into the probe is proportional to the spatial derivative of $\Delta T(r)$ at the surface of the probe. Accounting for the thermal conductivity of the brain and the geometry of the probe, this leads to an expression for the surface conductivity of an un-insulated probe, $\kappa_{S}$, expressed per unit length of the probe

$$
\kappa_{S}=\frac{2 \pi r_{P} \kappa_{B} K_{1}\left(r_{P} / \lambda\right)}{\lambda K_{0}\left(r_{P} / \lambda\right)}
$$


where $K_{1}$ is the first-order modified Bessel function of the second kind (the derivative of $\left.K_{0}\right)$.

In the most general case, some portion of the probe can be surrounded by several concentric layers of insulation. The conductivity of each insulating layer is derived via integration in cylindrical coordinates, which yields a logarithmic solution. Conductances of insulation layers act in series with the probe-brain interface conductance (Fig. 2G) and can therefore be represented by a single surface conductivity of the probe per unit length of the probe, $\kappa_{S}^{\prime}$ (to distinguish from $\kappa_{S}$ in the un-insulated case)

$$
\kappa_{S}^{\prime}=\left\lceil\sum_{i=1}^{N} \frac{\ln \left(r_{i} / r_{i-1}\right)}{2 \pi \kappa_{i}}+\frac{\lambda K_{0}\left(r_{N} / \lambda\right)}{2 \pi r_{N} \kappa_{B} K_{1}\left(r_{N} / \lambda\right)}\right]^{-1}
$$

Here $r_{1}, \ldots, r_{N}$ are the outer radii of the $N$ insulation layers, $\kappa_{1}, \ldots, \kappa_{N}$ are the material conductances of these layers, and $r_{0} \equiv r_{P}$. Note that the expression for the conductivity of the insulation-brain interface, the inverse of which is the second term of this equation, has been changed from Eqn. 16 to account for the increased radius of the interface. The ratio of $\kappa_{S}^{\prime}$ to this interface conductivity represents the efficiency of the insulation, expressing temperature at the surface of the insulation as a fraction of the probe temperature $\Delta T_{P}$ underneath the insulation. This fractional reduction in temperature is equivalent to the effect of a voltage divider in electrical circuits.

\subsection{Model of thermal conduction along a cylindrical probe in the brain}

In a typical cooling application, the probe in the brain would consist of two sections: an insulated segment of length $l_{1}$, and an un-insulated segment of length $l_{2}$ located deeper in the brain within the targeted area (a third section, located above the brain, will be considered later). We would like to determine how the probe temperature $\Delta T_{P}$ changes as a function of depth. Following the conventions of Cable Theory (Rall, 1962; Dayan and Abbott, 2001), we characterize the two segments of the probe by their length constants, $\lambda_{1}$ and $\lambda_{2}$, which represent how gradually temperature falls off along the length of the probe. Taking into account the geometry of the probe, we obtain

$$
\lambda_{1}=\sqrt{\frac{\pi r_{P}^{2} \kappa_{P}}{\kappa_{S}}}
$$

where $\kappa_{P}$ is the thermal conductivity of the probe material and $\kappa_{S}$ is the surface conductivity given by Eqn. 16. The length constant of the second segment, $\lambda_{2}$ is calculated using the same equation, but with the value of $\kappa_{S}^{\prime}$ given by Eqn. 17 instead of $\kappa_{S}$. Because the first segment has a lower surface conductivity due to insulation, it has a higher length constant than the second segment.

From Cable Theory, the general expression for temperature along the first segment, as a function of depth $x$ is

$$
\Delta T_{p}(X)=a_{1} \cosh \left(L_{1}-X\right)+b_{1} \sinh \left(L_{1}-X\right)
$$

where $L_{1} \equiv l_{1} / \lambda_{1}, X \equiv x / \lambda_{1}$, and $a_{1}$ and $b_{1}$ given by boundary conditions. Similarly, temperature along the second segment is 


$$
\Delta T_{P}(X)=a_{2} \cosh \left(L_{2}-X\right)+b_{2} \sinh \left(L_{2}-X\right)
$$

where $L_{2} \equiv l_{2} / \lambda_{2}, X \equiv x / \lambda_{2}$ (here, $x$ is measured from the upper end of the second segment rather than from the brain surface), and $a_{1}$ and $b_{1}$ are given by boundary conditions.

Boundary conditions require that both temperature and its derivative are continuous at the contact between the two segments. Further, we assume that no heat flows through the tip of the probe, implying that the derivative of temperature is 0 at the tip. (This is a reasonable boundary condition because the cross-sectional area of the tip is small relative to the overall exposed surface area). Using these considerations, we derive

$$
\begin{gathered}
a_{1}=\Delta T_{S}\left[\cosh L_{1}+\left(\lambda_{1} / \lambda_{2}\right) \sinh L_{1} \tanh L_{2}\right]^{-1} \\
b_{1}=\Delta T_{S}\left[\sinh L_{1}+\left(\lambda_{2} / \lambda_{1}\right) \cosh L_{1} \operatorname{coth} L_{2}\right]^{-1} \\
a_{2}=\Delta T_{S}\left[\cosh L_{1} \cosh L_{2}+\left(\lambda_{1} / \lambda_{2}\right) \sinh L_{1} \sinh L_{2}\right]^{-1} \\
b_{2}=0
\end{gathered}
$$

where $\Delta T_{S}$ is the temperature of the probe at the surface of the brain (the fourth boundary condition).

By taking the derivative at zero of the expression in Eqn. 19 and accounting for the probe conductivity, we can determine the input thermal conductance $K_{P}$ of the entire probe, measured at the surface of the brain:

$$
K_{P}=\frac{\pi r_{P}{ }^{2} \kappa_{P}}{\lambda_{1}}\left(\frac{1}{\operatorname{coth} L_{1}+\left(\lambda_{1} / \lambda_{2}\right) \tanh L_{2}}+\frac{1}{\tanh L_{1}+\left(\lambda_{2} / \lambda_{1}\right) \operatorname{coth} L_{2}}\right)
$$

As mentioned above, another segment of the probe, of length $l_{0}$, is located between the cold plate of the device and the brain surface. The thermal conductance of this segment acts in series with $K_{P}$ (Fig. 2G), allowing us to calculate the overall thermal load on the cold plate:

$$
K_{L}=K_{I}+N_{P}\left(\frac{l_{0}}{\pi r_{P}{ }^{2} K_{P}}+\frac{1}{K_{P}}\right)^{-1}
$$

where $K_{I}$ is the conductance of the cold-plate insulation discussed in Section 2.7.1 and $N_{P}$ is the number of probes attached to the cold plate (e.g., 2 for bilateral cooling experiments).

\section{Theoretical results: Optimization of the thermoelectric device}

Previous sections have derived equations that provide a detailed description of heat transfer and temperature changes in the thermoelectric device for brain cooling. Some aspects of this device are strongly constrained by an experiment (e.g., the necessary length of the probe in 
the brain). Other aspects, such as the shape of the semiconductor blocks or the probe diameter, can be chosen from a range of alternatives. In the following sections, we use the derived equations to characterize the dependence of device performance on various parameters of its construction. The goal is to learn how to optimize these parameters, as well as to gain a better understanding of experimental constraints on the device.

Here we discuss each parameter independently, showing the effect of changing a parameter on the performance of a "core" device, which contains two probes for bilateral cooling of a structure $2 \mathrm{~mm}$ deep in the brain. Various aspects of this core device are measured or approximated in Table 1. The construction of this device is described in Experimental Methods (Section 4.2) and the measurements of the parameters in Table 1 are discussed in Appendix A. Computer code for simulating device performance is provided in Supplementary Materials.

\subsection{Semiconductor geometry}

As mentioned earlier, a semiconductor block can be characterized by the geometric constant $G$, the ratio of its cross-sectional area to its height. Large values of $G$ are advantageous because they reduce Ohmic heating in the device. Small values of $G$, on the other hand, improve thermal isolation between the hot and cold plates of the device. Given these opposing influences, one would expect an optimal value of $G$ to exist for a given device.

Fig. 3A shows steady-state temperatures at the cold plate as a function of current for three devices with different values of $G$. As expected from the above derivations, temperature exhibits a minimum at an optimal value of the current for each configuration. Under the conditions shown here, the temperature at this minimum is the lowest for values of $G$ near $1.7 \mathrm{~mm}$. The optimal value of $G$ depends on other device parameters, such as the load on the cold plate and the efficiency of the heat sink. Fig. 3B shows minimum achievable temperatures for different loads in a reasonable range. Smaller loads allow lower temperatures to be achieved at the cold plate, and the optimal value of $G$ is somewhat lower for smaller loads. Efficiency of the heat sink also has a dramatic effect on the lowest achievable temperatures, as illustrated by increasing the thermal conductance of the convective heat sink, $K_{C}$ (Fig. 3C). Higher values of $G$ are optimal in the presence of an efficient heat sink, although the temperature minimum is fairly broad across values of $G$ in this case. In general, the consequences of having a $G$ that is slightly larger than optimal are less severe than having a $\mathrm{G}$ that is smaller than optimal. On the other hand, devices with larger $\mathrm{G}$ require more current to achieve a given minimum temperature (Fig 3A).

\subsection{Number of semiconductor junctions}

The device considered so far contains two semiconductor blocks, forming a single junction $(N=1)$. What is the effect of changing the number of junctions? For simplicity, consider increasing $N$ by a factor of 2 and simultaneously decreasing $G$ by the same factor. With this modification, the overall surface area of the semiconductor blocks does not change, leaving all thermal conductances in the device the same. For a given amount of current, heat production produced by the Peltier effect doubles due to increased number of junctions. Ohmic heat production, however, increases by a factor of 4 due to the combined effect of increasing the length of the current's path and decreasing the cross-sectional area of this path. Because the Peltier effect in linear and Ohmic heating is quadratic in current, these changes imply that equivalent temperature is achieved by the device at half the original amount of current. Fig. 3D confirms this reasoning: increasing $N$ and decreasing $G$ shifts the minimum of the temperature-current relationship to lower values of current, but leaves temperature at this minimum the same. 
Thus, increasing $N$ and leaving the product $N G$ at its optimal value does not improve or worsen device performance. However, it allows smaller currents to be used - a property advantageous for behavioral experiments on small animals, in which thin cables and small electrical commutator contacts must be used (Fee and Leonardo, 2001). The need to connect a large number of small semiconductor blocks can, in principle, make device construction difficult. However, some commercially-available thermoelectric modules have sizes and values of $G$ suitable for brain cooling in small animals (e.g., Custom Thermoelectric).

\subsection{Heat sinking}

The removal of heat from the hot plate of the Peltier device is necessary to achieve cooling of the cold plate. This heat arises from two sources: from heat pumped out of the brain during cooling and from Ohmic heating of the semiconductor elements. Heat sinking can be carried out using a number of approaches, the most common being water cooling. A water cooled heat sink requires that inlet and outlet flow tubing be supplied to the cooling device (Long and Fee, 2008), however such tubing can be a hindrance for experiments on small animals. Here we consider an alternative approach of using an air-cooled convective heat sink attached to the hot plate (see Experimental Methods).

This heat sink is constructed by attaching conductive "fins" to the hot plate, which are exposed to the air surrounding the animal (see Experimental Methods). In addition, the device includes a "body-couple heat sink," constructed by attaching a conducting material to the hot plate, and establishing thermal contact between that material and the surface of the animal's cranium. The convective and body-coupled heat sinks have thermal conductances $K_{C}$ and $K_{B}$, respectively.

Fig. 4A shows the maximum amount of cooling achievable by the device as a function of $K_{C}$ and $K_{B}$. Not surprisingly, our model suggests that increasing the conductivity of either heat sink generally improves device performance. An exception to this rule is that the bodycoupled heat sink slightly worsens performance when the convective heat sink is exceptionally efficient $\left(K_{C}>\sim 20 \mathrm{~mW} / \mathrm{K}\right)$. This occurs because such strong convection actually maintains the hot plate below body temperature, causing the body-coupled heat sink to pump heat into the plate, rather than away from it. Highly efficient convection can be achieved using active water cooling of the hot plate (Long and Fee, 2008). However, because we specifically set out to design a device without water cooling, our heat sinks are constrained to relatively small conductance values $\left(K_{B} \approx 7 \mathrm{~mW} / \mathrm{K}\right.$ and $K_{C} \approx 5 \mathrm{~mW} / \mathrm{K}$; see Methods). What are the effects of having either one of these heat sinks, and is including both of them in the device useful?

The effects of including or omitting each of the two heat sinks is illustrated in Fig 4B. Without either heat sink, the device does not generate cooling in steady-state at any amount of current (note that this device still produces a temperature differential between the hot and cold plates, but Ohmic heating keeps both plates above brain temperature). If either of the two heat sinks is included, the device is capable of generating some amount of cooling. The convective heat sink is more effective than the body-coupled one because it is linked to room temperature, which is about $18^{\circ} \mathrm{C}$ below body temperature.

The maximal cooling achieved by a device with both heat sinks is only marginally stronger than the maximal cooling achieved by the convective heat sink alone. However, these two devices are very different at zero current. Due to the exposure to room temperature, convective heat sinking alone cools the cold plate by almost $8^{\circ} \mathrm{C}$ (or $\sim 5^{\circ} \mathrm{C}$ in the targeted brain region $200 \mu \mathrm{m}$ from the probe) when no current is being passed. Including the bodycoupled heat sink roughly halves the amount of cooling at zero current to $\sim 4^{\circ} \mathrm{C}$ below body 
temperature at the cold plate (and only $\sim 2.5^{\circ} \mathrm{C}$ below body temperature in the targeted brain area).

\subsection{Probe dimensions}

So far, we have analyzed the effects of various device parameters on the temperature of the cold plate. If a probe is attached to the cold plate, however, the temperature change at the tip of this probe in the brain is less than that of the cold plate. This is primarily because the probe itself has finite axial conductance and some amount of heat is transferred through the imperfect insulation along the length of the probe. This has the effect of reducing the efficiency of the device at changing the temperature deep in the brain. These phenomena are dependent on the dimensions of the probe and the insulation, which we now consider.

Clearly, a poor thermal conductor must be selected for insulating the probes. Because air has very low thermal conductivity, we chose to insulate probes with polyimide tubes that were wide enough to leave some air between the probe surface and the polyimide wall (see Experimental Methods). Fig. 5A shows the simulated effect of such insulation on temperature in the brain near the probe. Not surprisingly, increasing the inner diameter of the polyimide tube improves insulation, as indicated by a greater fractional drop in temperature across the insulation. However, this improvement is small beyond a certain range of diameters (e.g. when increasing the inner diameter from 160 to $200 \mu \mathrm{m}$; Fig. 5A) and is made at the expense of increasing the outer diameter as well. Damage to brain tissue is a concern with large probes and should be taken in account when selecting insulation thickness, especially if increasing this thickness only marginally improves device performance.

If the inner and outer diameters of the polyimide tube are held fixed, what is the optimum choice of probe diameter? On the one hand, increasing the diameter reduces the thickness of the air insulation. On the other hand, it increases the cross-sectional area of the probe, improving axial conduction. These opposing effects suggest, and Figs. 5B,C confirm, the existence of an optimal probe diameter. For inner polyimide tube diameters in the range of several hundred microns, optimal cooling $(2 \mathrm{~mm}$ deep in the brain, $200 \mu \mathrm{m}$ away from the surface) is achieved by probes that fill approximately $2 / 3$ of the tube inner diameter; both thicker and thinner probes produce suboptimal cooling. Of course, it may sometimes be beneficial to choose a diameter smaller than the optimum in order to reduce the amount of tissue damage in the targeted brain region.

Finally, probe length is a parameter that cannot be optimized, since probes must be long enough to reach the region of interest. However, using a highly conductive material like silver and covering it with efficient insulation ensures that the length constant of the probe is long compared to the targeted depth. For the probes of our "core" device (see Experimental Methods), the length constant according to Eqn. 18 is $\lambda_{1}=72 \mathrm{~mm}$ for the insulated section and $\lambda_{2}=41 \mathrm{~mm}$ for the un-insulated section. Fig. 5D shows the amount of cooling achieved at the tip of the probe for various probe lengths. These calculations suggest that, for experiments that target deep structures (e.g. $8 \mathrm{~mm}$ ), the diameter of the probe and insulation may need to be larger than we used in our devices.

\section{Experimental Methods: Construction and testing of the devices}

We constructed two types of miniature devices for bilaterally cooling brain areas in the juvenile zebra finch (Fig. 1). One device was used for cooling nucleus HVC, which is located within 200 um of the brain surface, and thus uses a plate positioned over HVC in a planar geometry. The other device was used for cooling nucleus LMAN (lateral magnocellular nucleus of the anterior nidopallium). LMAN is located $\sim 2 \mathrm{~mm}$ deep in the 
brain and was cooled by inserting thin cylindrical wires into the nucleus. This device was analyzed using cylindrical geometry.

\subsection{Construction of the devices}

We constructed a small Peltier thermoelectric device using commercially-available bismuthtelluride $\left(\mathrm{Bi}_{2} \mathrm{Te}_{3}\right)$ semiconductor elements (Custom Thermoelectric). These elements were trimmed using a razor blade to a $1.5 \times 1.5 \mathrm{~mm}$ cross-sectional area, $1.35 \mathrm{~mm}$ height, achieving an approximate value of $G=1.7 \mathrm{~mm}$. A single p-type and a single n-type element ( $N=1$ junction) were soldered to metalized pads on a thermally-conductive ceramic "hot plate" $(8 \times 8 \mathrm{~mm})$ using bismuth-tin solder (Custom Thermoelectric). The distance between the two elements was $\sim 1 \mathrm{~mm}$. Note that complete miniature thermoelectric modules with larger values of $N$ and similar values of the product $N G$ are also commercially available (e.g., Custom Thermoelectric) and can be used instead of this process. This allows the use of smaller currents to achieve optimal cooling (see Section 3.2).

To deliver current to the device, we soldered a miniature connector (Omnetics) to the hot plate, making electrical contacts with the two semiconductor elements. For HVC cooling (Fig. 1A-C), a thin annealed silver sheet $(1 \times 10 \mathrm{~mm}, 5$ mil thick) was soldered to the bottom of the semiconductor blocks. The sheet was gold-plated (cyanide-free gold-plating solution; Neuralynx) to prevent silver toxicity, and bent to form two cooling pads $(1 \times 2 \mathrm{~mm})$ with 3 $\mathrm{mm}$ spacing between the inner edges.

For LMAN cooling (Fig. 1D-F), a small stainless-steel "cold plate" was soldered to the bottom of the semiconductor blocks. Two sharpened gold-plated annealed silver wires (250 $\mu \mathrm{m}$ diameter, $3.5 \mathrm{~mm}$ long) were soldered to this cold plate, forming the "probes" that were inserted bilaterally into both LMANs. These wires were thermally insulated with polyimide tubing ( 1 mil-thick walls), with an inner diameter of $320 \mu \mathrm{m}$, leaving $35 \mu \mathrm{m}$ of air between the wire and the inner surface of the polyimide tube. The last $1 \mathrm{~mm}$ of each wire was left uninsulated, and the bottom tip of each polyimide tube was sealed with a drop of a silicone elastomer (KwikCast from WPI). The un-insulated segments of the wires were inserted bilaterally into LMAN.

For both HVC and LMAN cooling devices, the semiconductor blocks and the cold plate were insulated with a $1 \mathrm{~mm}$-thick layer of Styrofoam, which was further coated with a thin layer of KwikCast for moisture sealing. For both devices, a convective heat sink was constructed by soldering a copper wire mesh ( 2 mil wires, 200 threads per inch; from TWP) around the perimeter of the hot plate, extending $8 \mathrm{~mm}$ above the device. Thin silver strips were soldered to the edges of the mesh (above the corners of the hot plate) for structural support, as well as to improve thermal conduction away from the hot plate. At two locations, the top of the mesh was also glued to the connector to improve structural stability.

A body-coupled heat sink was constructed from a 5-mil annealed silver sheet, which was soldered to the hot plate on one end. At the other end, the sheet was cut longitudally into 1 $\mathrm{mm}$-thin strips. These strips were bent to follow the curvature of the skull, forming contact with a total surface area of $\sim 6 \times 8 \mathrm{~mm}$. The space between the silver and the skull surface ( $200 \mu \mathrm{m}$ thickness) was filled with light-cured dental acrylic (Pentron Clinical) to facilitate heat transfer. Note that acrylics that use methyl methacrylate are not compatible with this device because methyl methacrylate fumes dissolve Styrofoam insulation.

\subsection{Surgery}

Subjects were juvenile zebra finches (male or female, 35-50 days-post-hatch). Birds were obtained from the Massachusetts Institute of Technology breeding facility. Animal care and 
experiments were carried out in accordance with the National Institute of Health guidelines and approved by the local Institutional Animal Care and Use Committee.

Birds were anesthetized and placed in a stereotaxic apparatus. Craniotomies were made bilaterally, and HVC or LMAN were targeted using stereotaxic coordinates. For LMAN cooling, small $(\sim 400 \times 400 \mu \mathrm{m})$ craniotomies were made above LMAN and probes were slowly inserted into the brain, advancing by $100-200 \mu \mathrm{m}$ per minute. Probe tips were implanted $2.5 \mathrm{~mm}$ deep, such that the un-insulated segments at the tip of the probes spanned the dorso-ventral extent of LMAN (1.5 $-2.5 \mathrm{~mm}$ deep). For HVC cooling, $1 \times 2 \mathrm{~mm}$ windows of the skull over the two HVCs were thinned using a dental drill, and the cooling pads of the device were placed in contact with these windows.

\subsection{Measurement of brain temperature changes}

To determine the effect of the thermoelectric devices on brain temperature, measurements were made with thin (40-gauge) thermocouples (Omega) implanted in the brain at several distances from the cooling plate in HVC-cooled birds, and at several distances from the cooling probes in LMAN-cooled birds. Because isoflurane anesthesia produces a substantial decrease in brain temperature, temperature measurements to characterize the performance of the devices were carried out in awake animals. The measurements were done within several hours of the surgery.

Following surgery, birds were placed into a foam restraint and allowed to recover from anesthesia until fully conscious ( $10 \mathrm{~min})$. Various levels of stable DC current (see text) were passed through the device using a current source (ZUP60-14 from Lambda). Temperature measurements were acquired at 100 samples/s and recorded with customwritten software in Matlab. Following temperature measurements, birds were sacrificed and the distance between the device and the thermocouple was verified by taking $\mathrm{X}$-ray images of the intact skull at several angles, showing the cooling device and thermocouples.

Although temperature measurements were carried out on the day of the surgery, we used devices for HVC and LMAN cooling chronically in juvenile zebra finches to study changes in the forebrain control of song timing. These experiments were performed up to 1 month following surgery. During this period, birds produced normal amounts of singing, and we typically did not observe any problems associated with device implantation. The observed behavioral effects of brain cooling had similar magnitudes throughout this period (Aronov and Fee, unpublished observations).

\section{Experimental Results: Performance of the implemented devices}

In the following sections, we characterize temperature changes produced by these devices in awake juvenile birds using small thermocouples implanted in the brain or attached to the cooling devices themselves.

\subsection{Device for HVC cooling}

Our device achieves HVC cooling via metal plates pressed against the surface of the brain. To characterize the performance of this device, we measured temperatures of these cooling plates at various amounts of electric current in 3 birds. In 2 of these birds, we also measured temperature near the center of $\mathrm{HVC}$, about $500 \mu \mathrm{m}$ below the cooling plates in the brain. Fig. 6A shows temperature measurements at these two locations. For each amount of current, we estimated the steady-state temperature (Fig. 6B) by fitting an exponential to measurements in the last $70 \mathrm{~s}$ of the corresponding current pulse. 
As expected, convective heat loss from the device produced slight cooling even at zero current. At about $0.5 \mathrm{~A}$, however, heating maintained the cooling plates and $\mathrm{HVC}$ close to normal body temperature $\left(41^{\circ} \mathrm{C}\right)$. Maximum cooling of roughly $10^{\circ} \mathrm{C}$ at the cooling plate was achieved at about $-1.5 \mathrm{~A}$. At the center of HVC, temperature changes were roughly $3 / 4$ of those at the cooling plate. Given a distance of $500 \mu \mathrm{m}$, this fraction is accurately predicted by an exponential fall-off with a space constant of $\lambda=1.59 \mathrm{~mm}$ (Section 2.7.1).

For some experiments it may useful to alternate between warm and cooled conditions. To illustrate this mode of operation, we alternated the current between 0.5 and $-1.5 \mathrm{~A}$ every $100 \mathrm{~s}$. Fig. $6 \mathrm{C}$ shows temperature changes produced by this protocol. Following a change in current, temperature in this experiment typically achieved values close to the steady-state within 10-20 s. At currents of $-1.5 \mathrm{~A}$ and above, temperature following these initial changes was relatively stable, typically drifting by less than $1^{\circ} \mathrm{C}$ during a 100 -s current pulse. The total range of temperatures was about $10^{\circ} \mathrm{C}$ at the cooling plate and about $7^{\circ} \mathrm{C}$ in the center of HVC. To ensure that temperature has reached values close to the steady state, it may be desirable to only use data recorded during the last $80 \mathrm{~s}$ of each current pulse.

\subsection{Device for LMAN cooling}

In 4 additional birds, we measured temperature changes produced by the device for LMAN cooling. LMAN is located $\sim 2 \mathrm{~mm}$ deep in the brain and is therefore cooled by implanting thermally conductive probes into the brain (see Experimental Methods). Fig. 6D,E show temperature measurements at the cold plate of the device, at the tip of the probe, and at about $500 \mu \mathrm{m}$ from the surface of the probe in the brain. Timecourses of temperature changes were similar to those produced by the HVC cooling device described above. At the cold plate, maximal cooling by roughly $15^{\circ} \mathrm{C}$ was achieved at currents near $-1.5 \mathrm{~A}$. As expected, temperature changes at the tip of the probe were smaller, resulting in roughly $11^{\circ} \mathrm{C}$ of cooling. These values are remarkably similar (within $1^{\circ} \mathrm{C}$ ) to those predicted by the thermal model of the "core" device described in the previous section. Also according to expectations, temperature fall-off in cylindrical coordinates was substantially greater than in the planar coordinates - about $4^{\circ} \mathrm{C}$ of cooling were achieved at $500 \mu \mathrm{m}$ from the surface of the probe in the brain.

Because brain temperature changes substantially with distance from a cooling probe, average amount of cooling achieved in LMAN depends on the exact positioning of the probes within the nucleus. Therefore, it is important to precisely characterize this spatial relationship. We measured temperature at various distances of up to $1 \mathrm{~mm}$ from the probes. Fig. $6 \mathrm{~F}$ shows temperature measurement at three of the locations, during the current protocol described above (alternating between 0.5 and -1.5 A every $100 \mathrm{~s}$ ). About $10^{\circ} \mathrm{C}$ of cooling were achieved near the probe, but temperature fell off steeply with increasing distances - to about half this amount at $300 \mu \mathrm{m}$ from the probe. The fall-off became more gradual beyond this range; about $3^{\circ} \mathrm{C}$ of cooling were achieved at $1 \mathrm{~mm}$ from the probe. These values were close to those predicted by the thermal diffusion model in cylindrical coordinates described above (Section 2.7.2).

\section{Discussion}

Characterization of temperature changes in the brain produced by thermoelectric devices poses several challenges, due to the number of physical processes involved in this technique. In this study, we provided a simple mathematical model that accounts for these processes. Although heat transfers in Peltier devices and along insulated wires (Rowe, 1995), as well as in the brain (Kastella and Fox, 1971; Jafari and Higgins, 1989), have been modeled before, here we have combined relevant aspects of these models into a single thermal-circuit framework that allows temperature changes in the brain to be accurately simulated (see 
equations in the text and the computer code in Supplementary Material). This framework is useful for characterizing device performance and for optimizing various parameters of its construction, since the effect of any parameter on temperature can be predicted.

Using this theoretical framework, we demonstrated the design and construction of a miniature device for cooling both superficial and deep brain areas in a small songbird. A crucial feature of this device, in comparison with previous versions, is that it generates sufficient temperature changes without a water-cooled heat sink or any other attached tubing. Rather, unwanted heat in this device is removed using convective air cooling. This feature substantially simplifies device construction and, more importantly, allows brain cooling to be used in a wider range of unrestrained animal behaviors. The device was sufficiently lightweight to be used in juvenile zebra finches, which weigh only $\sim 12 \mathrm{~g}$, and therefore should be usable in most vertebrate animal models. Although we successfully used this device for cooling LMAN, an area $2 \mathrm{~mm}$ deep in the brain with a diameter of roughly $800 \mu \mathrm{m}$, cooling larger and deeper structures may require using probes with a larger diameter (see Section 3.4).

What can we learn from cooling areas in the brain? Because temperature changes specifically alter the speeds of biophysical processes in a relatively small region, this technique offers a unique way of targeting behaviorally-relevant biophysical dynamics. In particular, this method is effective in a typical feedforward motor system, where an oscillator circuit projects onto a premotor area, which then converts the oscillatory signal into a sequence of motor commands (Stent et al., 1978). Cooling the oscillator circuit slows down its biophysical dynamics, resulting in an equivalent slowing of the behavior itself (Bauer and von Helversen, 1987; Pires and Hoy, 1992). On the other hand, cooling of the premotor area downstream of the oscillator may be somewhat disruptive to the behavior, but cannot change its speed. A recent study in the zebra finch illustrates the power of this technique in a complex, vertebrate motor system that is homologous to the mammalian cortex: Whereas cooling nucleus HVC uniformly slows down the song, cooling its efferent nucleus RA has no effect on song speed, suggesting that the dynamics intrinsic to $\mathrm{HVC}$ are actively involved in the control of song timing (Long and Fee, 2008).

Importantly, the distinction of a brain area that is simply necessary for a behavior from one that actually generates the behaviorally-relevant dynamics is often difficult or impossible with the more traditional techniques. Because both such brain areas are necessary for the production of a movement, lesioning or inactivating either one can be equally disruptive to the behavior. Similarly, global stimulation of either brain area can disrupt the behavior, particularly if feedback connections are present between them (Ashmore et al., 2005). Finally, neuronal recordings in either brain area can reveal similar premotor activity patterns time-locked to the rhythmic behavioral output (Stent et al., 1978; Chi and Margoliash, 2001; Hahnloser et al., 2002).

More broadly speaking, a critical question in neuroscience is how the biophysical dynamics intrinsic to neural circuits produce activity patterns like oscillations (Buzsáki, 2006), persistent activity (Goldman-Rakic, 1995; Major and Tank, 2004), neuronal sequences (Abeles, 1991; Hahnloser et al., 2002; Pastalkova et al., 2008), and stochastic processes (van Vreeswijk and Sompolinsky, 1998; Vogels et al., 2005; Wilson, 1999). Such processes are thought to underlie a variety of internal brain functions, but their origins are often poorly understood, especially in complex circuits with multiple interconnected brain areas. In these cases, cooling may be an efficient technique for localizing the biophysical dynamics that generate intrinsic activity patterns in the brain. 


\section{Supplementary Material}

Refer to Web version on PubMed Central for supplementary material.

\section{Appendix A}

\section{Measurement of semiconductor properties}

To estimate properties of semiconductor elements we constructed a device without cooling pads or probes, but with Styrofoam insulation covering the cold plate (Fig. A1a). We placed the hot plate in contact with the surface of a large water bath to maintain it at near-constant temperature and used two thermocouples to simultaneously monitor the temperatures of the hot and cold plates. We varied the current flow $I$ through the device from -10 to $5 \mathrm{~A}$ in steps of $0.1 \mathrm{~A}$ and measured the temperature difference $\Delta T$ between the hot and cold plates. We also used an instrumentation amplifier to measure the voltage drop across the two semiconductor elements of the device. The current steps were applied for $90 \mathrm{~s}$; steady-state voltage and temperatures were calculated by taking the average in the last $60 \mathrm{~s}$ of each current pulse.

At currents near zero, the voltage across the device changed linearly with current (Fig. A1b). We estimated the slope of this relationship in the temperature range relevant for our experiments $\left(25-42^{\circ} \mathrm{C}\right.$ of the average temperature between the hot and cold plates). Using this slope, we estimated the electrical resistivity $\rho$ in this temperature range to be about 1740 $\mu \Omega \mathrm{cm}$. According to Eqn. 5, we fit a quadratic function to the relationship between $\Delta T$ and $I$ (Fig. A1c). Using the coefficients of this fit, we estimated the Seebeck coefficient of the semiconductor material to be $148 \mu \mathrm{V} / \mathrm{K}$ and the thermal conductivity to be $1.38 \mathrm{~W} \mathrm{~m}^{-1} \mathrm{~K}^{-1}$. The estimated value of the Seebeck coefficient is somewhat lower (by $\sim 25 \%$ ) than the values typically reported in the literature (Rowe, 1995;Kondratiev and Yershova, 2001); the estimated value of the electrical resistivity is about $50 \%$ higher. These differences could be due to the influences of some aspects of our device construction (e.g. the solder joints) on performance.

\section{Measurement of heat sink conductances}

We sought to roughly estimate the thermal conductances $K_{B}$ of the body-coupled heat sink and $K_{C}$ of the convective heat sink in our device (Fig. 2E). For this, we constructed devices identical to our normal devices, but with no probes or cooling pads attached, and with surface-mount resistors (total resistance $R=10 \Omega$ ) soldered to the hot plate instead of the similarly-sized semiconductor elements. For any given current $I$ passed through the device, this allowed us to estimate the total power $\left(P=I^{2} R\right)$ dissipated by the device. Resistors were insulated with a 1-mm thick layer of Styrofoam. Devices were attached to the cranium in 3 birds and a thermocouple (see above) was used to monitor the temperature of the hot plate. Brain and air temperatures were simultaneously monitored with two additional thermocouples. These latter two temperatures remained roughly constant throughout the experiment $\left(\sim 41^{\circ} \mathrm{C}\right.$ for the brain, $\sim 23^{\circ} \mathrm{C}$ for air).

We measured the temperature drop $\Delta T_{1}$ from the hot plate to the air and the drop $\Delta T_{2}$ from the hot plate to the brain. For a given amount of power $P$ generated by current flow through the device, the equivalent thermal circuit (Fig. 2E) implies the relationship $P=\Delta T_{1} K_{C}+$ $\Delta T_{2} K_{B}$. We took two measurements - one at zero current (generating $P=0 \mathrm{~mW}$ ) and one at a current generating $P=200-250 \mathrm{~mW}$ of power - and used this relationship to solve for $K_{B}$ and $K_{C}$. (In all cases, the temperature of the hot plate remained below $45^{\circ} \mathrm{C}$ ). In the 3 birds, values of $K_{B}$ were 9.2, 6.9, and 7.4 milliwatts per Kelvin $(\mathrm{mW} / \mathrm{K})$, and values of $K_{C}$ were 
6.2, 4.8, and $5.1 \mathrm{~mW} / \mathrm{K}$. For the simulation of our "core" device, we used approximate median values $K_{B}=7 \mathrm{~mW} / \mathrm{K}$ and $K_{C}=5 \mathrm{~mW} / \mathrm{K}$.

\section{Acknowledgments}

We acknowledge helpful conversations with Michael Long. Funding for this work was provided by a National Institutes of Health grant (R01 DC009183). Additional support to DA was provided by a Hertz Foundation Silvio Michali Fellowship.

\section{References}

Abeles, M. Corticonics. Cambridge University Press; 1991.

Arbas EA, Calabrese RL. Rate modification in the heartbeat central pattern generator of the medicinal leech. J Comp Physiol A. 1984; 155:783-794.

Ashmore RC, Wild JM, Schmidt MF. Brainstem and forebrain contributions to the generation of learned motor behaviors for song. J. Neurosci. 2005; 25:8543-8554. [PubMed: 16162936]

Bauer M, von Helversen O. Separate localization of sound recognizing and sound producing neural mechanisms in a grasshopper. J Comp Physiol A. 1987; 161:95-101.

Bech C, Midtgård U. Brain temperature and therete mirabile ophthalmicum in the Zebra finch (Poephila guttata). J Comp Physiol B. 1981; 145:89-93.

Bonifazi P, Goldin M, Picardo MA, Jorquera I, Cattani A, Bianconi G, Represa A, Ben-Ari Y, Cossart R. GABAergic hub neurons orchestrate synchrony in developing hippocampal networks. Science. 2009; 326:1419-1424. [PubMed: 19965761]

Bowman, F. Introduction to Bessel functions. Courier Dover Publications; 1958.

Buzsáki, G. Rhythms of the brain. US: Oxford University Press; 2006.

Chafee MV, Goldman-Rakic PS. Inactivation of Parietal and Prefrontal Cortex Reveals Interdependence of Neural Activity During Memory-Guided Saccades. J Neurophysiol. 2000; 83:1550-1566. [PubMed: 10712479]

Chi Z, Margoliash D. Temporal precision and temporal drift in brain and behavior of zebra finch song. Neuron. 2001; 32:899-910. [PubMed: 11738034]

Dayan, P.; Abbott, LF. Theoretical Neuroscience. MIT Press; 2001.

Duck, FA. Physical properties of tissue. Academic Press; 1990.

Fee MS, Leonardo A. Miniature motorized microdrive and commutator system for chronic neural recording in small animals. J Neurosci Methods. 2001; 112:83-94. [PubMed: 11716944]

Feng AS. The effect of temperature on a social behavior of weakly electric fish Eigenmannia virescens. Comp Biochem Physiol A: Comp Physiol. 1976; 55:99-102. [PubMed: 7423]

Ferster D, Chung S, Wheat H. Orientation selectivity of thalamic input to simple cells of cat visual cortex. Nature. 1996; 380:249-252. [PubMed: 8637573]

Foster JA, Robertson RM. Temperature Dependency of Wing-Beat Frequency in Intact and Deafferented Locusts. J Exp Biol. 1992; 162:295-312.

Gerhardt HC. Temperature Coupling in the Vocal Communication System of the Gray Tree Frog, Hyla versicolor. Science. 1978; 199:992-994. [PubMed: 17752373]

Goldman-Rakic PS. Cellular basis of working memory. Neuron. 1995; 14:477-485. [PubMed: 7695894]

Goller F, Cooper BG. Peripheral motor dynamics of song production in the zebra finch. Ann. N. Y. Acad. Sci. 2004; 1016:130-152. [PubMed: 15313773]

Hahnloser RHR, Kozhevnikov AA, Fee MS. An ultra-sparse code underlies the generation of neural sequences in a songbird. Nature. 2002; 419:65-70. [PubMed: 12214232]

Harris-Warrick RM, Cohen AH. Serotonin modulates the central pattern generator for locomotion in the isolated lamprey spinal cord. J. Exp. Biol. 1985; 116:27-46. [PubMed: 4056654]

Imoto H, Fujii M, Uchiyama J, Fujisawa H, Nakano K, Kunitsugu I, Nomura S, Saito T, Suzuki M. Use of a Peltier chip with a newly devised local brain-cooling system for neocortical seizures in the rat. Technical note. J. Neurosurg. 2006; 104:150-156. [PubMed: 16509160] 
Jafari F, Higgins PD. Thermal modeling in cylindrical coordinates using effective conductivity. IEEE Trans Ultrason Ferroelectr Freq Control. 1989; 36:191-196. [PubMed: 18284967]

Kastella KG, Fox JR. The Dynamic Response of Brain Temperature to Localized Heating. Biophys J. 1971; 11:521-539. [PubMed: 5558703]

Katz PS, Sakurai A, Clemens S, Davis D. Cycle Period of a Network Oscillator Is Independent of Membrane Potential and Spiking Activity in Individual Central Pattern Generator Neurons. J Neurophysiol. 2004; 92:1904-1917. [PubMed: 15115787]

Kondratiev, D.; Yershova, L. TE coolers computer simulation: incremental upgrading of rate equations approach; Proceedings of the Sixth European Workshop on Thermoelectricity of the European Thermoelectric Society; 2001.

Lashley, KS. The problem of serial order in behavior. In: Jeffress, LA., editor. Cerebral Mechanisms in Behavior. Wiley; 1951. p. 112-136.

Levelt, WJM. Speaking: from intention to articulation. MIT Press; 1993.

Lomber SG, Payne BR, Horel JA. The cryoloop: an adaptable reversible cooling deactivation method for behavioral or electrophysiological assessment of neural function. J. Neurosci. Methods. 1999; 86:179-194. [PubMed: 10065985]

Long MA, Fee MS. Using temperature to analyse temporal dynamics in the songbird motor pathway. Nature. 2008; 456:189-194. [PubMed: 19005546]

Major G, Tank D. Persistent neural activity: prevalence and mechanisms. Curr. Opin. Neurobiol. 2004; 14:675-684. [PubMed: 15582368]

Pastalkova E, Itskov V, Amarasingham A, Buzsáki G. Internally generated cell assembly sequences in the rat hippocampus. Science. 2008; 321:1322-1327. [PubMed: 18772431]

Pires A, Hoy RR. Temperature coupling in cricket acoustic communication. II. Localization of temperature effects on song production and recognition networks in Gryllus firmus. J. Comp. Physiol. A. 1992; 171:79-92. [PubMed: 1403993]

Rall W. Theory of physiological properties of dendrites. Ann. N. Y. Acad. Sci. 1962; 96:1071-1092. [PubMed: 14490041]

Rowe, DM. CRC handbook of thermoelectrics. CRC Press; 1995.

Stent GS, Kristan WB, Friesen WO, Ort CA, Poon M, Calabrese RL. Neuronal generation of the leech swimming movement. Science. 1978; 200:1348-1357. [PubMed: 663615]

Tang LS, Goeritz ML, Caplan JS, Taylor AL, Fisek M, Marder E. Precise temperature compensation of phase in a rhythmic motor pattern. PLoS Biol. 20108 Available at: http:// www.ncbi.nlm.nih.gov/pubmed/20824168.

Tanji J. Sequential organization of multiple movements: involvement of cortical motor areas. Annu. Rev. Neurosci. 2001; 24:631-651. [PubMed: 11520914]

Thompson SM, Masukawa LM, Prince DA. Temperature dependence of intrinsic membrane properties and synaptic potentials in hippocampal CA1 neurons in vitro. J Neurosci. 1985; 5:817-824. [PubMed: 3973697]

Tryba AK, Peña F, Lieske SP, Viemari J, Thoby-Brisson M, Ramirez J. Differential modulation of neural network and pacemaker activity underlying eupnea and sigh-breathing activities. J. Neurophysiol. 2008; 99:2114-2125.

Vogels TP, Rajan K, Abbott LF. Neural network dynamics. Annu. Rev. Neurosci. 2005; 28:357-376. [PubMed: 16022600]

Volgushev M, Vidyasagar TR, Chistiakova M, Eysel UT. Synaptic transmission in the neocortex during reversible cooling. Neuroscience. 2000a; 98:9-22. [PubMed: 10858607]

Volgushev M, Vidyasagar TR, Chistiakova M, Yousef T, Eysel UT. Membrane properties and spike generation in rat visual cortical cells during reversible cooling. J. Physiol. (Lond.). 2000b; 522(Pt. 1):59-76. [PubMed: 10618152]

van Vreeswijk C, Sompolinsky H. Chaotic balanced state in a model of cortical circuits. Neural Comput. 1998; 10:1321-1371. [PubMed: 9698348]

Wilson, HR. Spikes, decisions, and actions. Oxford University Press; 1999.

Yamaguchi A, Gooler D, Herrold A, Patel S, Pong WW. Temperature-Dependent Regulation of Vocal Pattern Generator. J Neurophysiol. 2008; 100:3134-3143. [PubMed: 18829853] 
Yu AC, Margoliash D. Temporal hierarchical control of singing in birds. Science. 1996; 273:18711875. [PubMed: 8791594]

Zhang JX, Ni H, Harper RM. A miniaturized cryoprobe for functional neuronal blockade in freely moving animals. J. Neurosci. Methods. 1986; 16:79-87. [PubMed: 3702491] 


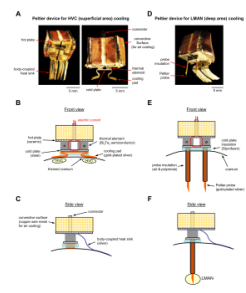

Figure 1. Implementation of two Peltier-based thermoelectric devices for localized brain cooling (A) Photograph of the device for cooling a structure located close to the surface of the brain (nucleus HVC in the songbird) using cooling pads placed against thinned areas of the cranium. (B-C) Schematics of the device shown in (a). (D) Photograph of the device for cooling a structure deep in the brain (nucleus LMAN in the songbird) using thermally conductive probes. (E-F) Schematics of the device shown in (D). Abbreviations: HVC, used as a proper name, formerly high vocal center; LMAN, lateral magnocellular nucleus of the nidopallium. 
A

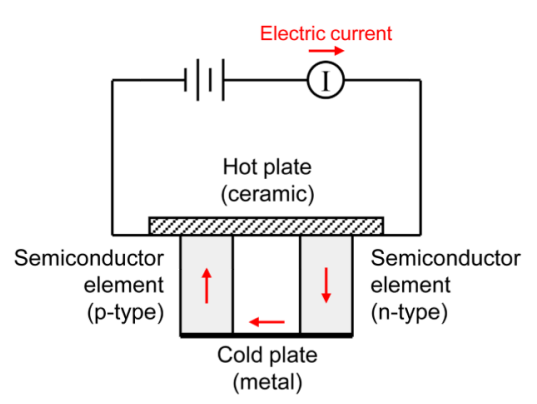

D

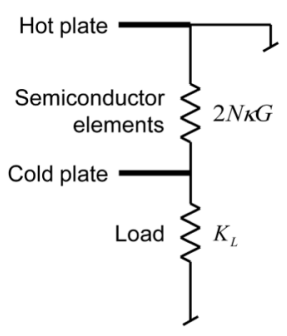

$\mathbf{F}$

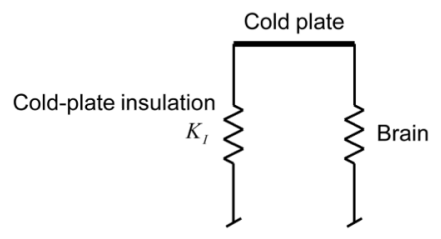

B

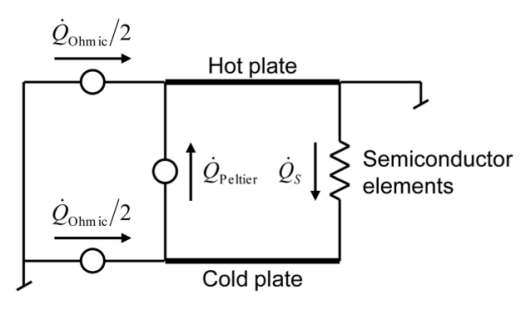

C

Cold-plate temperature

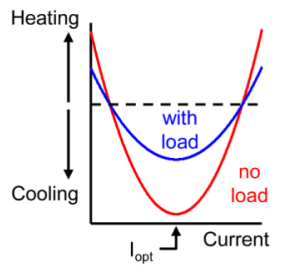

$\mathbf{E}$

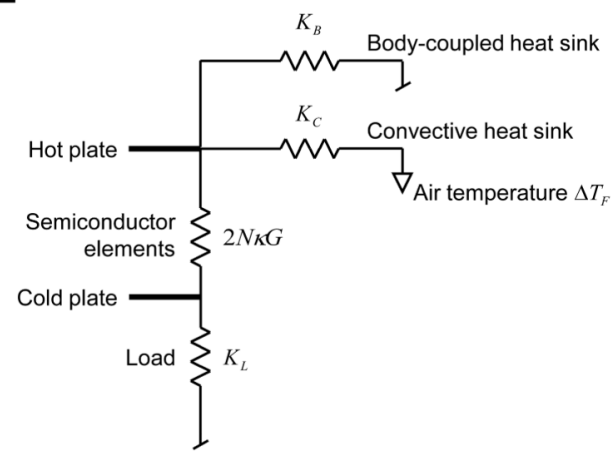

G

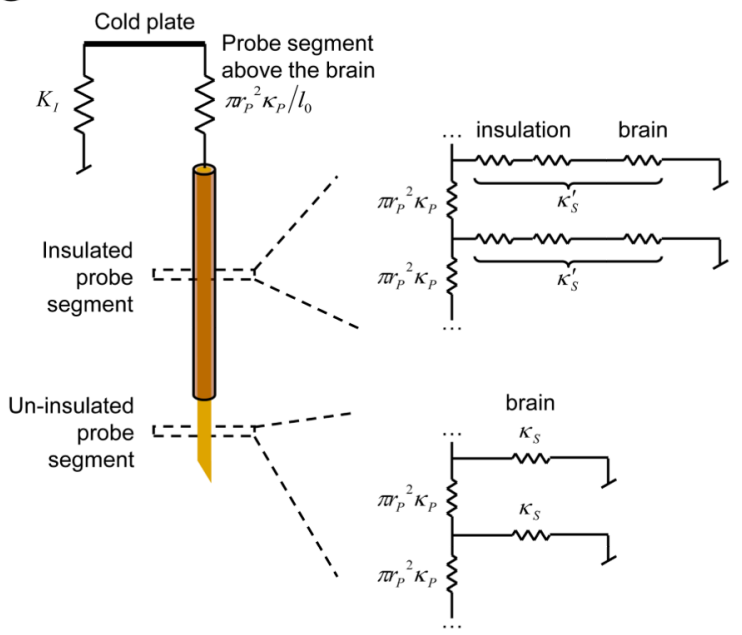

Figure 2. Analysis of the thermoelectric device using equivalent thermal circuits

(A) Schematic of a device. Electric current flows back-and-forth between the hot and the cold plates along semiconductor elements. For one direction of current flow, the Peltier effect cools the cold plate and heats up the hot plate. One semiconductor pair $(\mathrm{N}=1)$ is shown. The device is attached to a perfect heat sink, represented by "grounding" the hot plate. (B) Equivalent thermal circuit for the device shown in (A). Semiconductor elements are represented by a thermal conductance. Heat is pumped from the cold plate due to the Peltier effect $\left(\dot{Q}_{\text {Peltier }}\right)$. Ohmic heat from the current flow $\left(\dot{Q}_{\text {Ohmic }}\right)$ is transferred equally to each of the plates. (C) Plot of the steady-state temperature achieved at the cold plate for an isolated device and for the same device attached to a thermal load. Optimal cooling is 
achieved at current $I_{\text {opt }}$. (D) Equivalent circuit for a device attached to a load, represented by a conductance $K_{L}$. (E) Equivalent circuit of a device with non-ideal heat sinks represented by conductances: $K_{B}$ is a body-coupled heat sink; $K_{C}$ is a convective heat sink, cooled by a fluid of temperature $\Delta T_{F}$ relative to body temperature. (F) Equivalent circuit of the load on the cold plate in the planar geometry. Insulation is represented by a thermal conductance $K_{I}$ in parallel with the brain. $(\mathrm{G})$ Equivalent circuit of the load on the cold plate in cylindrical geometry. Only one probe is shown. The probe has axial thermal conductivity (vertical resistors) that depends on its radius $r_{P}$ and material conductivity $\kappa_{P}$. Segments within the brain also have substantial surface conductivity $\kappa_{S}$ for the un-insulated segment and $\kappa_{S}^{\prime}$ for the insulated one), expressed per unit length of the probe. 


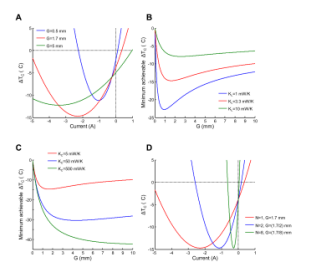

Figure 3. Influence of semiconductor geometry on device performance

(A) Steady-state temperature of the cold plate $\Delta T_{C}$ as a function of current flow through the device, for three values of the geometric constant $G$. Geometric constant of the semiconductor elements affect the minimum-achievable temperature. (B) Minimum achievable $\Delta T_{C}$ as a function of $G$, for different values of the thermal load. For the load conductance estimated for our device (3.3 milliwatts per Kelvin, $\mathrm{mW} / \mathrm{K}$ ), the lowest temperature is achieved for $G \approx 1.7 \mathrm{~mm}$ (red trace). Note that lower values of $G$ are optimal for smaller loads. (C) Relationship between the minimum achievable $\Delta T_{C}$ and $G$ for different conductances of the convective heat sink, $K_{C}$. Larger values of $G$ are optimal for more efficient heat sinks. (D) Relationship between $\Delta T_{C}$ and current for three devices with different numbers of semiconductor junctions $(N)$. For each device, the value of $G$ is scaled by $N$ such that the overall surface area of the semiconductors remains the same. Devices with more semiconductor junctions achieve identical temperatures at the cold plate, but less current is required to achieve the minimum temperature. 
A

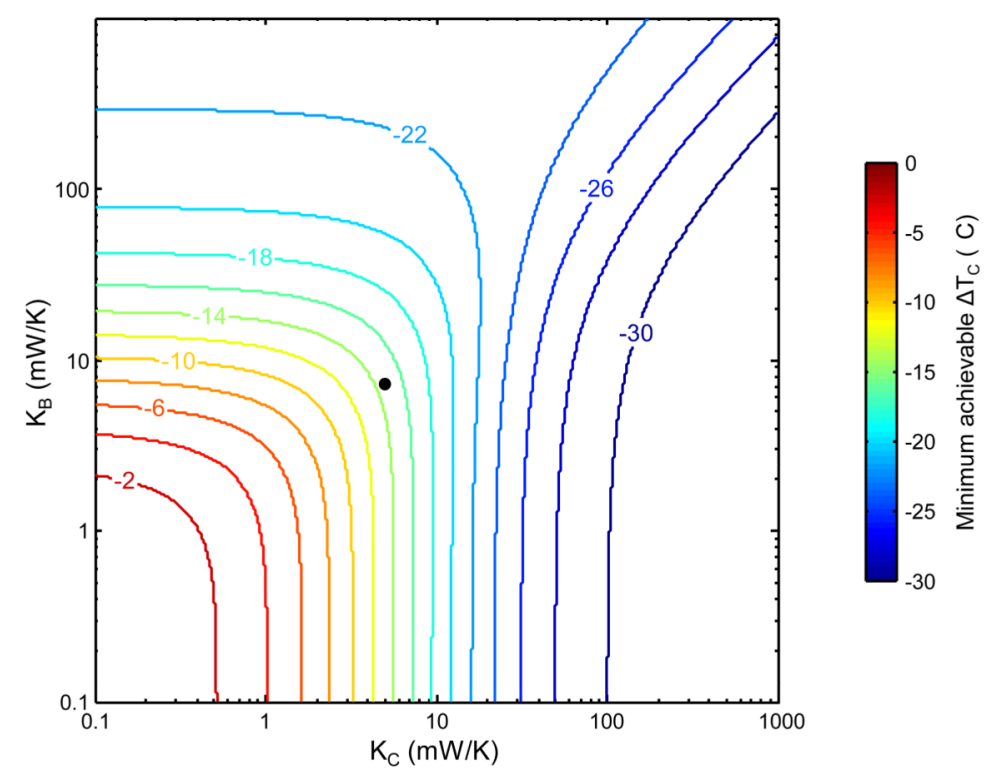

B

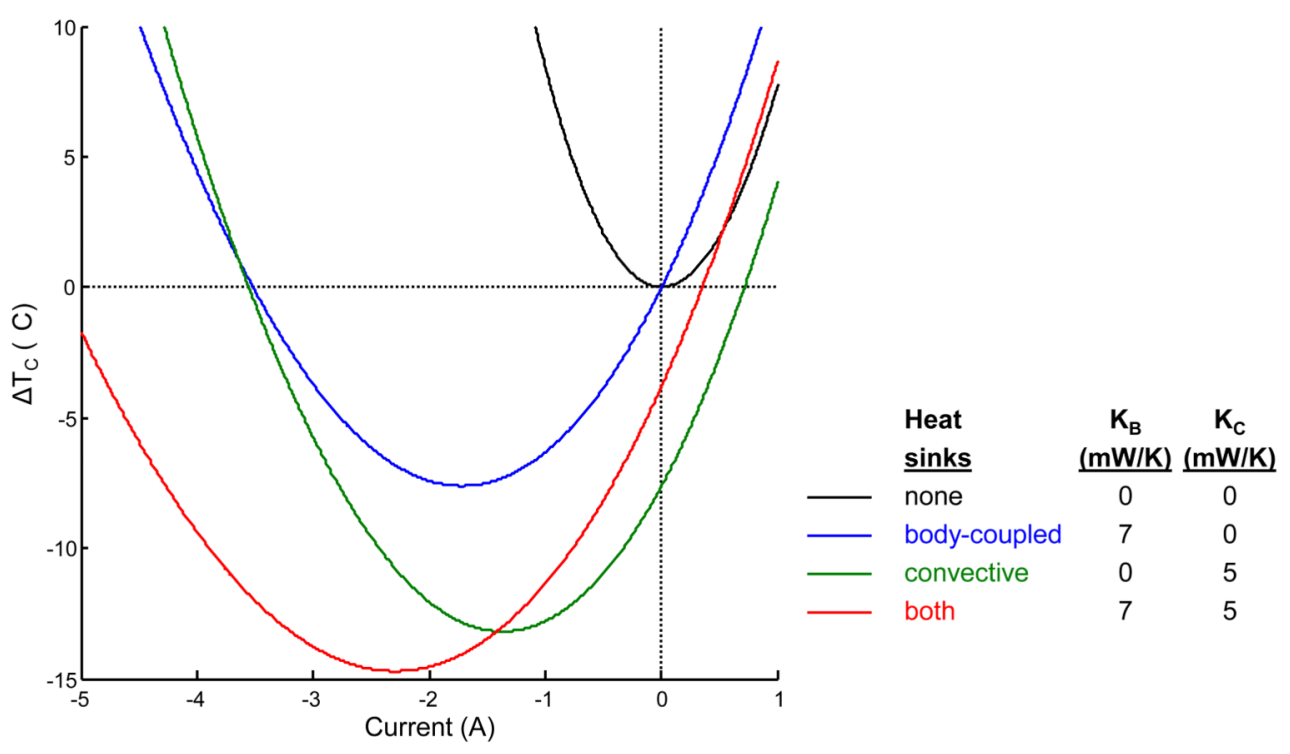

Figure 4. Influence of heat sinks on device performance

(A) Minimum steady-state temperature achievable at the cold plate, $\Delta T_{C}$ as a function of two heat sink conductances: the body-coupled heat sink $K_{B}$ and the convective heat sink $K_{C}$. At small conductance values, improving either heat sink improves the minimum achievable temperature at the cold plate. However, when the convective heat sink is efficient $(>\sim 20$ milliwatts per Kelvin, $\mathrm{mW} / \mathrm{K}$ ), attaching a body-coupled heat sink does not improve performance. Contours are spaced at intervals of $2^{\circ} \mathrm{C}$; numbers indicate $\Delta T_{C}$ in ${ }^{\circ} \mathrm{C}$. Black symbol indicates heat sink values of our "core" device (see text). (B) Effects of including or omitting small body-coupled $(7 \mathrm{~mW} / \mathrm{K})$ and convective $(5 \mathrm{~mW} / \mathrm{K})$ heat sinks. For each combination of heat sinks, the steady-state temperature at the cold plate is shown as a 
function of device current. The lowest temperature is achievable when both heat sinks are included (red trace). Including both heat sinks, in addition to improving the maximum cooling of the device compared convective heat sink alone (green trace), substantially reduces the amount of unwanted cooling at zero current. 


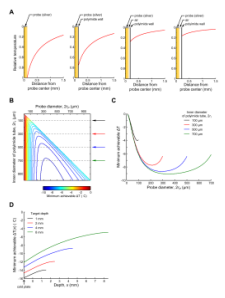

Figure 5. Optimizing probe insulation for cooling deep brain structures

(A) Effectiveness of various probe insulations in the brain. Leftmost plot shows the spatial profile (red trace) of the steady-state temperature in the brain near an un-insulated probe. The three rightmost plots shown this profile for probes insulated with a polyimide tube (25 $\mu \mathrm{m}$-thick wall), with different amounts of air insulation $(0,35$, and $75 \mu \mathrm{m})$ between the probe and the polyimide tube. (B) Minimum temperature achievable in the brain $(2 \mathrm{~mm}$ deep, $200 \mu \mathrm{m}$ from the central axis of the probe), for different dimensions of the probe and the insulation. For each configuration, the polyimide wall is $25 \mu \mathrm{m}$ thick and the space between the probe surface and the inner polyimide wall is filled with air. Contours are spaced at intervals of $0.5^{\circ} \mathrm{C}$. (C) Effects of varying probe diameter while holding the polyimide tube diameter fixed. Traces are shown for four different diameters of polyimide tube. For each polyimide tube diameter, an optimum probe diameter exists. (D) Temperature profile along the length of the probe, for various probe lengths. For each probe, the initial 1$\mathrm{mm}$ segment is above the brain (and effectively ideally insulated by being embedded in Styrofoam) and the final 1-mm segment is un-insulated, such that the targeted depth is at the center of the un-insulated segment. The rest of the probe is insulated with polyimide tubing. 


\section{HVC cooling}

A

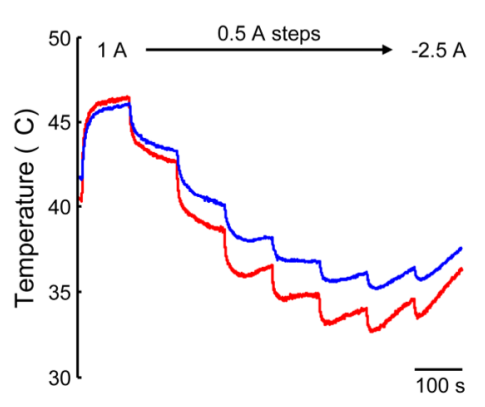

$B$

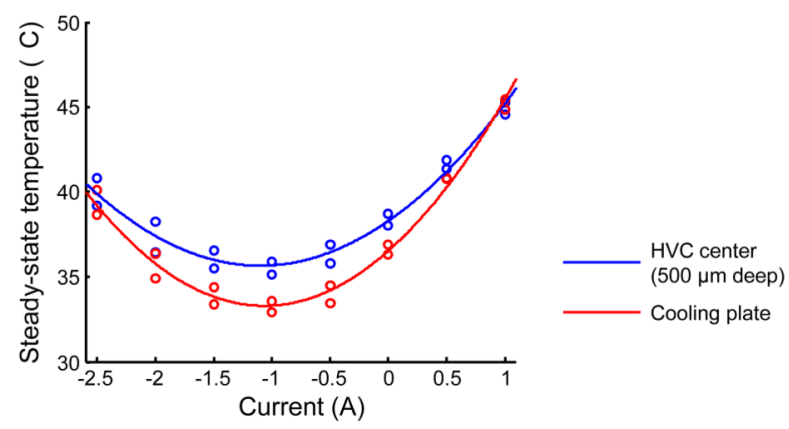

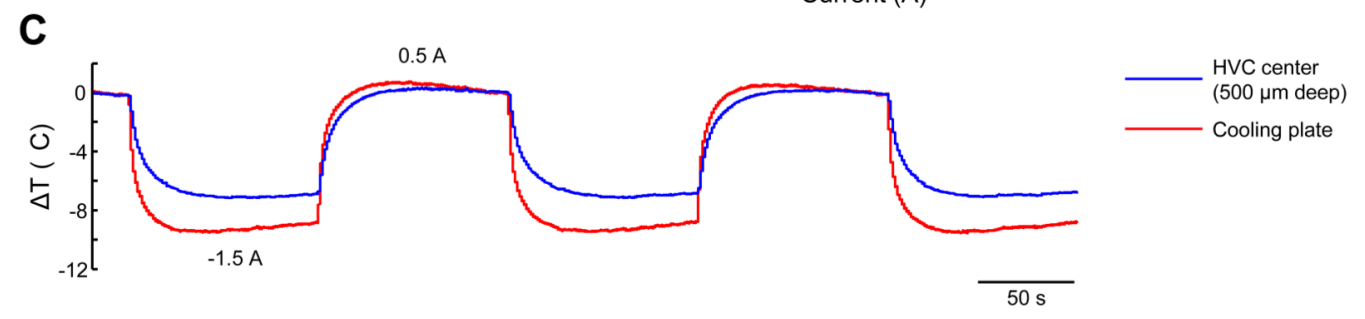

LMAN cooling

D
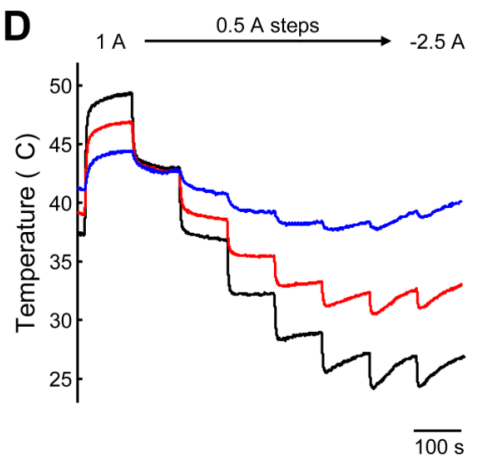

E

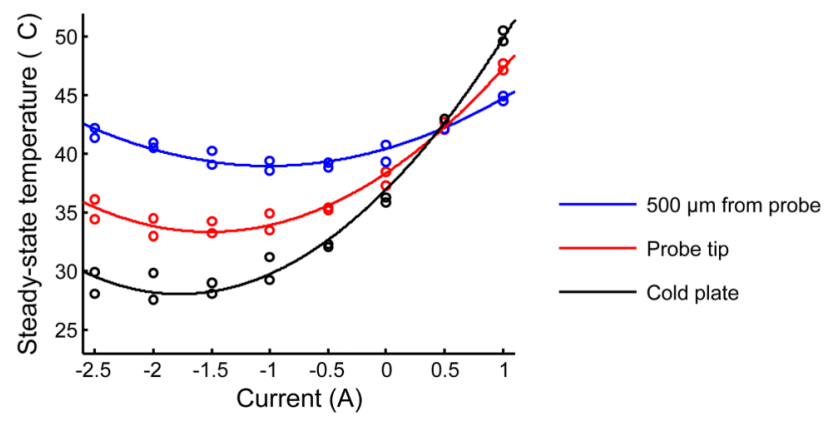

F

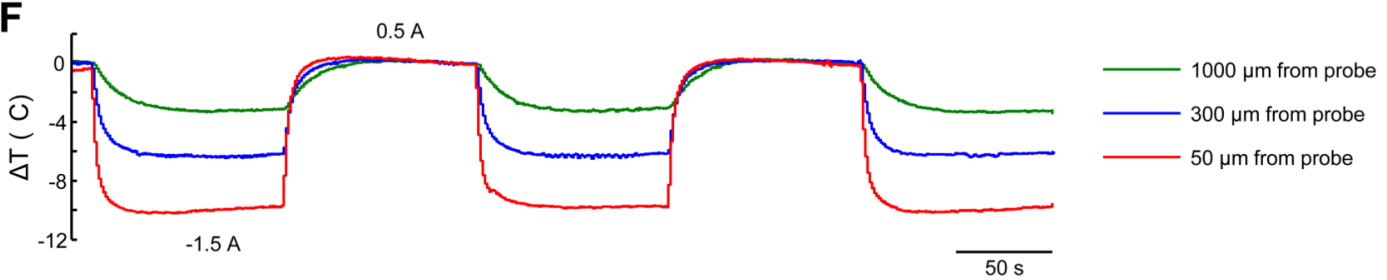

Figure 6. Measurements of temperature produced by thermoelectric devices

(A) Temperature at the cooling plate and in $\mathrm{HVC}$ at various amounts of current flown through the HVC cooling device. The amount of current is changed every $100 \mathrm{~s}$, decreasing from 1 to $-2.5 \mathrm{~A}$ in steps on $0.5 \mathrm{~A}$. (B) Steady-state temperature as a function of current at the two locations. Steady-state is estimated by fitting an exponential curve to the last $70 \mathrm{~s}$ of each current step in (A). (C) Temperature at the same two locations during an experiment in which current is alternated between 0.5 and -1.5 A every $100 \mathrm{~s}$. (D,E) Temperatures at the cold plate of the LMAN cooling device, at the probe tip, and at $500 \mu \mathrm{m}$ away from the surface of the probe. Data are estimated and plotted as in (A,B). (F) Temperature at three 
distances from the probe surface of the LMAN cooling device during alternation of the current between 0.5 and -1.5 A every $100 \mathrm{~s}$, as in (C). 
A

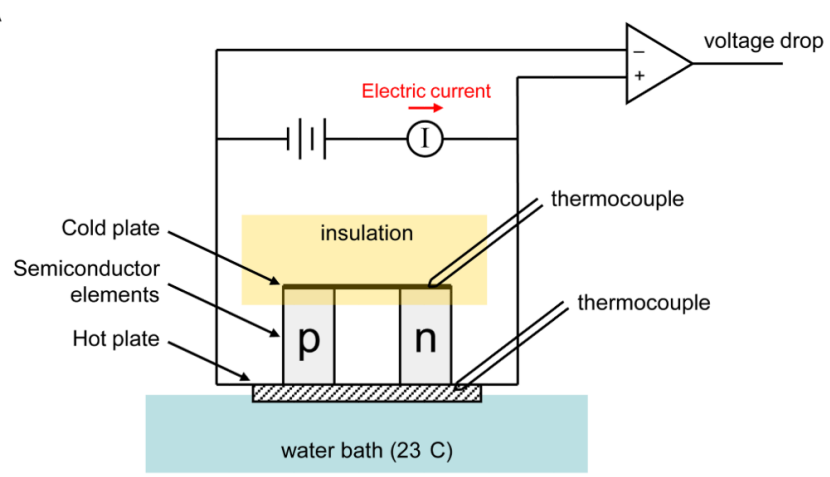

B

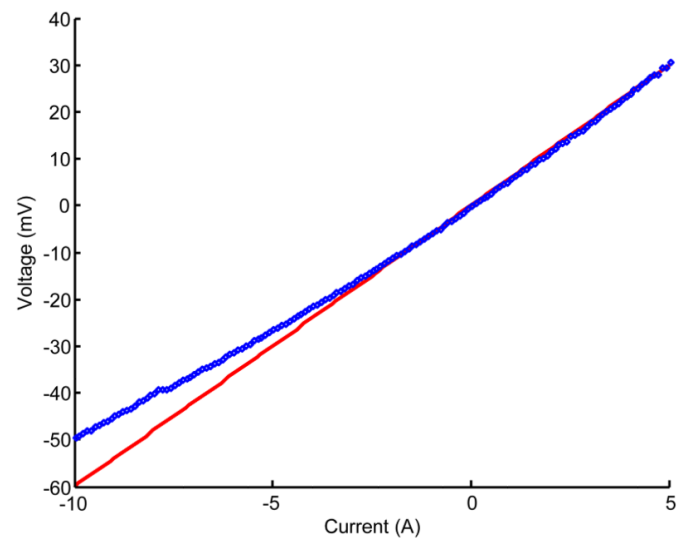

C

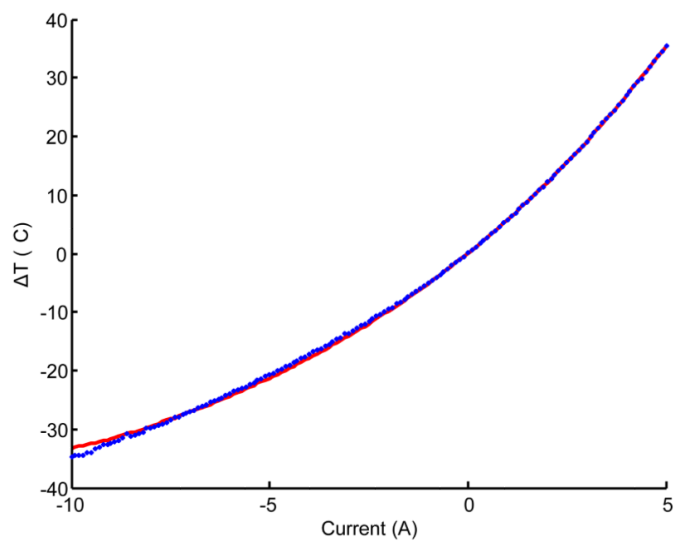

Figure A1. (In Appendix A) Estimation of semiconductor properties

(A) Schematic of the experimental setup. The hot plate of the Peltier device is maintained at a near-constant temperature using a large water bath; the cold-plate is insulated with Styrofoam. The temperature difference, $\Delta T$, between the hot and cold plates is measured with small thermocouples. A constant current pulse ( $90 \mathrm{~s}$ duration) is applied to the device, and an instrumentation amplifier is also used to record the voltage drop across the semiconductor elements. (B) Average steady-state voltage across the device at different values of current (blue symbols). The red line is a linear fit to the data in the range from $\left(25-42^{\circ} \mathrm{C}\right)$. Note that the data deviate from the linear fit, likely due to the temperaturedependence resistance of the semiconductor material. (C) Average steady-state temperature 
difference between the hot and the cold plates at different current levels (blue symbols). A quadratic fit to the data is shown (red curve), from which the Seebeck coefficient and thermal conductivity of the semiconductor elements were estimated. 


\section{Table 1}

Various parameters of the "core" device analyzed in the text. The device is used for cooling LMAN - a structure $\sim 2 \mathrm{~mm}$ deep in the brain. Units for conductances $\left(K_{B}, K_{C}\right.$, and $\left.K_{I}\right)$ are milliwatts per Kelvin (mW/K). See text for details.

\begin{tabular}{|c|c|c|c|}
\hline Parameter & Description & Value & Comment \\
\hline \multicolumn{4}{|c|}{ Semiconductor properties } \\
\hline$\alpha$ & Seebeck coefficient & $148 \mu \mathrm{V} \mathrm{K}^{-1}$ & $\begin{array}{l}\text { Measured (see } \\
\text { Methods) }\end{array}$ \\
\hline$\rho$ & electrical resistivity & $1740 \mu \Omega \mathrm{cm}$ & $\begin{array}{l}\text { Measured (see } \\
\text { Methods) }\end{array}$ \\
\hline$\kappa$ & thermal conductivity & $1.38 \mathrm{~W} \mathrm{~m}^{-1} \mathrm{~K}^{-1}$ & $\begin{array}{l}\text { Measured (see } \\
\text { Methods) }\end{array}$ \\
\hline$G$ & geometric constant & $1.7 \mathrm{~mm}$ & $\begin{array}{l}\text { Dimensions }(1 \times w \times h) \text { : } \\
1.5 \times 1.5 \times 1.35 \mathrm{~mm}\end{array}$ \\
\hline$N$ & number of junctions & 1 & $\begin{array}{l}\text { Two blocks (one each } \\
\text { of } n \text { - and p-type) }\end{array}$ \\
\hline \multicolumn{4}{|c|}{ Heat sink properties } \\
\hline$K_{B}$ & thermal conductance (body-coupled heat sink) & $7 \mathrm{~mW} \mathrm{~K}^{-1}$ & $\begin{array}{l}\text { Measured (see } \\
\text { Methods) }\end{array}$ \\
\hline$K_{C}$ & thermal conductance (convective heat sink) & $5 \mathrm{~mW} \mathrm{~K}^{-1}$ & $\begin{array}{l}\text { Measured (see } \\
\text { Methods) }\end{array}$ \\
\hline$\Delta T_{F}$ & temperature of convective fluid relative to body & $-18^{\circ} \mathrm{C}$ & $\begin{array}{l}\text { Air at room } \\
\text { temperature }\left(23^{\circ} \mathrm{C}\right)\end{array}$ \\
\hline \multicolumn{4}{|c|}{ Probe properties } \\
\hline$r_{P}$ & Radius & $125 \mu \mathrm{m}$ & \\
\hline$l_{0}$ & length of segment above the brain & $1.0 \mathrm{~mm}$ & \\
\hline$l_{1}$ & length of insulated segment & $1.5 \mathrm{~mm}$ & \\
\hline$l_{2}$ & length of un-insulated segment & $1.0 \mathrm{~mm}$ & $\begin{array}{l}\text { Center of segment is } \\
2.0 \mathrm{~mm} \text { deep }\end{array}$ \\
\hline$\kappa_{P}$ & thermal conductivity of material (silver) & $429 \mathrm{~W} \mathrm{~m}^{-1} \mathrm{~K}^{-1}$ & \\
\hline$N_{P}$ & number of probes & 2 & For bilateral cooling \\
\hline \multicolumn{4}{|c|}{ Insulation properties } \\
\hline$K_{I}$ & thermal conductance of cold-plate insulation & $0.17 \mathrm{~mW} \mathrm{~K}^{-1}$ & $\begin{array}{l}\text { For Styrofoam sheet: } \\
5 \mathrm{~mm}^{2} \text { area, } 1 \mathrm{~mm} \text { thick }\end{array}$ \\
\hline$r_{1}$ & outer radius of 1st insulation layer (air) & $160 \mu \mathrm{m}$ & \\
\hline$\kappa_{1}$ & thermal conductivity of air & $0.025 \mathrm{~W} \mathrm{~m}^{-1} \mathrm{~K}^{-1}$ & \\
\hline$r_{2}$ & outer radius of 2nd insulation layer (polyimide) & $185 \mu \mathrm{m}$ & \\
\hline$\kappa_{2}$ & thermal conductance of polyimide & $0.12 \mathrm{~W} \mathrm{~m}^{-1} \mathrm{~K}^{-1}$ & \\
\hline \multicolumn{4}{|c|}{ Brain properties } \\
\hline$\lambda$ & length constant & $1.59 \mathrm{~mm}$ & From data in (Long \& Fee 2008) \\
\hline$\kappa_{B}$ & thermal conductivity & $0.54 \mathrm{~W} \mathrm{~m}^{-1} \mathrm{~K}^{-1}$ & (Duck 1990) \\
\hline$T_{B}$ & baseline temperature & $314 \mathrm{~K}$ & (Bech \& Midtgård 1981) \\
\hline
\end{tabular}

